\title{
Effect of seasonal malaria chemoprevention plus azithromycin on Plasmodium falciparum transmission: gametocyte infectivity and mosquito fitness
}

Koudraogo Bienvenue Yaméogo $0^{1,2^{*}}$, Rakiswendé Serge Yerbanga ${ }^{1,3}$, Seydou Bienvenu Ouattara ${ }^{1}$, Franck A. Yao ${ }^{1}$, Thierry Lefèvre ${ }^{1,4,5,6}$, Issaka Zongo ${ }^{1}$, Frederic Nikièma' ${ }^{1}$, Yves Daniel Compaoré1, Halidou Tinto ${ }^{7}$, Daniel Chandramohan ${ }^{8}$, Brian Greenwood ${ }^{8}$, Adrien M. G. Belem², Anna Cohuet ${ }^{4,5}$ and Jean Bosco Ouédraogo ${ }^{1,3}$

\begin{abstract}
Background: Seasonal malaria chemoprevention (SMC) consists of administration of sulfadoxine-pyrimethamine (SP) + amodiaquine (AQ) at monthly intervals to children during the malaria transmission period. Whether the addition of azithromycin (AZ) to SMC could potentiate the benefit of the intervention was tested through a double-blind, randomized, placebo-controlled trial. The effect of SMC and the addition of $A Z$, on malaria transmission and on the life history traits of Anopheles gambiae mosquitoes have been investigated.

Methods: The study included 438 children randomly selected from among participants in the SMC $+A Z$ trial and 198 children from the same area who did not receive chemoprevention. For each participant in the SMC + AZ trial, blood was collected 14 to 21 days post treatment, examined for the presence of malaria sexual and asexual stages and provided as a blood meal to An. gambiae females using a direct membrane-feeding assay.

Results: The SMC treatment, with or without AZ, significantly reduced the prevalence of asexual Plasmodium falciparum $\left(L R T X_{2}^{2}=69, P<0.0001\right)$ and the gametocyte prevalence $\left(L R T X_{2}{ }_{2}=54, P<0.0001\right)$. In addition, the proportion of infectious feeds ( $\left(R T X_{2}^{2}=61, P<0.0001\right.$ ) and the prevalence of oocysts among exposed mosquitoes (LRT $X_{2}^{2}=22.8, P<0.001$ ) was reduced when mosquitoes were fed on blood from treated children compared to untreated controls. The addition of AZ to SPAQ was associated with an increased proportion of infectious feeds $\left(L R T X^{2}{ }_{1}=5.2\right.$, $P=0.02)$, suggesting a significant effect of AZ on gametocyte infectivity. There was a slight negative effect of SPAQ and SPAQ + AZ on mosquito survival compared to mosquitoes fed with blood from control children $\left(\mathrm{LRTX}^{2}{ }_{2}=330\right.$, $P<0.0001)$.
\end{abstract}

Conclusion: This study demonstrates that SMC may contribute to a reduction in human to mosquito transmission of P. falciparum, and the reduced mosquito longevity observed for females fed on treated blood may increase the benefit of this intervention in control of malaria. The addition of AZ to SPAQ in SMC appeared to enhance the infectivity of gametocytes providing further evidence that this combination is not an appropriate intervention.

\footnotetext{
*Correspondence: yamkbienvenue@yahoo.fr

${ }^{1}$ Institut de Recherche en Sciences de la Santé, Bobo-Dioulasso, Burkina Faso

Full list of author information is available at the end of the article
}

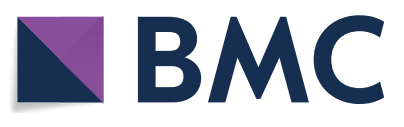

(c) The Author(s) 2021. Open Access This article is licensed under a Creative Commons Attribution 4.0 International License, which permits use, sharing, adaptation, distribution and reproduction in any medium or format, as long as you give appropriate credit to the original author(s) and the source, provide a link to the Creative Commons licence, and indicate if changes were made. The images or other third party material in this article are included in the article's Creative Commons licence, unless indicated otherwise in a credit line to the material. If material is not included in the article's Creative Commons licence and your intended use is not permitted by statutory regulation or exceeds the permitted use, you will need to obtain permission directly from the copyright holder. To view a copy of this licence, visit http://creativecommons.org/licenses/by/4.0/. The Creative Commons Public Domain Dedication waiver (http://creativeco mmons.org/publicdomain/zero/1.0/) applies to the data made available in this article, unless otherwise stated in a credit line to the data. 
Keywords: Seasonal malaria chemoprevention, Azithromycin, Gametocytes, Transmission

\section{Background}

Malaria remains a major cause of morbidity and mortality, particularly in Africa where $94 \%$ of cases occur. Effective management of clinical cases associated with the use of insecticide-impregnated material has been effective in reducing malaria transmission and has greatly contributed to the progresses in the fight against the disease since the beginning of the century. However, the reduction of malaria burden has stalled, or even reversed, in some countries in the recent years and additional strategies are needed. These include chemopreventive strategies such as intermittent preventive treatment during pregnancy (IPTp) and seasonal malaria chemoprevention (SMC) for children [1]. SMC consists of administration of therapeutic doses of sulfadoxine-pyrimethamine (SP) + amodiaquine (AQ) at monthly intervals to children aged 3-59 months during the peak malaria transmission period in areas where malaria transmission is seasonal [2]. In 2019, 21.5 million children in 13 countries in Africa's Sahel and sub-Sahel sub-region received malaria prophylaxis through SMC programmes [1]. SMC proved to be safe and cost-effective when deployed at the population level [3]. Other advantages of SMC are that it relies on frequent interactions between health staff or training volunteers and young children, which provides an opportunity for further benefits for populations, such as preventive administration of other drugs [4]. Mass administration of azithromycin (AZ) has been reported to provide a reduction in all cause mortality [5, 6]. Therefore, to determine whether the addition of $\mathrm{AZ}$ to SMC could reduce child mortality and morbidity was tested through a double-blind, randomized, placebocontrolled trial in young children in Burkina Faso and Mali. This trial showed that the addition of AZ reduced the incidence of several infectious diseases but did not reduce the incidence of deaths or hospital admissions [4]. Addition of AZ to SMC had little effect on the incidence of clinical attacks of malaria, but it may have had some effect on malaria transmission, and determining whether this was the case warrants further attention.

The transmission of malaria parasites from human to mosquito vector requires the presence of mature Plasmodium gametocytes in the ingested blood and both host and parasite factors can influence the production of gametocytes and their infectivity to mosquitoes [7, 8]. A large panel of drugs, including anti-malarial molecules, show positive or negative influence on gametocytogenesis, on gametocyte infectivity, and/or on parasite development in the mosquito [9-11] and SMC, with or without additional drugs, is therefore expected to have an effect on human to mosquito transmission.

$\mathrm{SP}$, and AQ to a lesser extent, have been shown to enhance the number of gametocytes circulating in the peripheral blood [12-14], possibly due to the release of sequestered parasites or through natural periodicity of parasite development [15]. However, the infectivity of gametocytes following SP treatment was low [16, 17]. In addition, SP has been shown to have a deleterious effect on mosquito survival [17], reflecting the complex effects of drug administration on malaria transmission. Mass administration of antibiotics to humans may affect the mosquito microbiota and possibly influence malaria parasite transmission in this way. Indeed, gut-inhabiting bacteria have been shown to interfere with parasite transmission in the mosquito and to modulate vector competence [18-24]. For instance, the presence of Enterobacteriaceae, such as Serratia marcescens, reduced the prevalence and intensity of infection [20] or even 
conferred refractoriness [20, 21, 24-26]. Experimental administration of antibiotics to mosquitoes either facilitated or impeded their infection, which suggested that antibiotics circulating in the blood of human hosts may impact the susceptibility of blood-sucking Anopheles gambiae females to transmit malaria infection by disturbing their gut microbiota [27]. Moreover, other parameters influencing vector capacity, such as mosquito lifespan and fecundity, are also affected by ingestion of antibiotics [28]. AZ reduced the Plasmodium falciparum gametocyte exflagellation process [29] and negatively affected the $P$. falciparum infection load as well as the mosquito lifespan [28] suggesting a potential for AZ to reducing malaria transmission.

The present study aimed at evaluating the effect of SMC with or without the addition of AZ on malaria transmission. It relied on a clinical trial that evaluated the benefit of adding AZ to SPAQ [4] and assessed the effect of SPAQ and SPAQ + AZ on the transmissibility of $P$. falciparum and on the life history traits of An. gambiae mosquitoes.

\section{Methods \\ Study site}

The study was conducted in Houndé health district, located about $100 \mathrm{~km}$ from Bobo-Dioulasso along the paved road to Ouagadougou, Burkina Faso. In this area, the rainy season lasts approximately six months from May to October. The main malaria vectors are reported to be Anopheles gambiae, Anopheles coluzzii [30]; Anopheles arabiensis is a secondary vector in the area [31]. Plasmodium falciparum is the most prevalent malaria parasite [32].

Child mortality caused by malaria remains high in Houndé in 2013 (36-40\%) [33]. Malaria transmission occurs during and shortly after the rainy season. Treatment based on artemisinin combination therapy (ACT) and bed-net coverage are major malaria control interventions prior to the study. SMC or mass administration of $\mathrm{AZ}$ was not deployed at this site prior the study, but now the site areas receive SMC provided by the national malaria control programmes [4].

In the SMC + AZ trial, the percentage of children who received at least three directly observed cycles of the assigned regimen was 86.8 and $84.3 \%$, respectively in 2015 and 2016 [4].

The population was estimated at 329,162 inhabitants in the Houndé health district in 2019 and is divided into villages over an area of $5622 \mathrm{sq} \mathrm{km} \mathrm{[34,} \mathrm{35].} \mathrm{Four} \mathrm{villages:}$ Koumbia, Dougmato, Kari and Boni, among the sites included in the SMC + AZ trial were selected randomly for this study.
Four month, each year, during the peak malaria transmission, study participants received SMC during 3 days. In addition, they received $\mathrm{AZ}$ or matching $\mathrm{AZ}$ placebo. All treatment doses were based on age and administered by study staff. The village of Pè in the district of Houndé, where SMC was not yet in place, was selected as a control.

\section{Study participants}

The study participants were age 24-59 months at the start of the study. For ethical consideration, subjects aged 3-24 months from whom venous blood collection may often present difficulties did not select. Sample size was estimated on the basis of calculations for comparing SMC alone versus $\mathrm{SMC}+\mathrm{AZ}$, and $\mathrm{SMC}$ versus no SMC. Based on the weekly survey during the first year of the study, assuming that with SMC to have about $5 \%$ of mosquitoes infected, and in control children, about $15 \%$ or more of mosquitoes infected; if $\mathrm{AZ}$ reduces mosquito infectivity by $50 \%$, to have $80 \%$ power to detect a difference, in total at least 20 to 30 children in each group, with 50 mosquitoes fed on each child would be needed.

In the four treated villages involved in the $\mathrm{SMC}+\mathrm{AZ}$ trial [4], 438 participants were randomly selected from among the 21,737 participants for inclusion in the transmission study. The inclusion criteria were age 24-59 months and parental or other legal guardian consent. Exclusion criteria were chronic illness, symptomatic malaria, planned long-term absence from the village during the study period, or absence at the moment of inclusion, difficulty in collecting venous blood, or retraction of consent. In the control group village of Pê, 198 participants were selected according to the same inclusion/ exclusion enrolment criteria used in the villages where chemoprevention was provided. Information about the trial recruitment was provided during meetings held in the medical centre. Ethical approval for the study was obtained from the national ethic committee of Burkina Faso under the Registration No. 2015-5-56.

\section{Treatment}

For the participants who were part of the SMC + AZ trial, anti-malarials were administered by trial staff monthly between August and November according to the agenda of the trial and to be in the line with the annual peak of malaria transmission season [4].

Children received $500 \mathrm{mg}$ of SP and $25 \mathrm{mg}$ of pyrimethamine plus $150 \mathrm{mg}$ of AQ on day 1 and $150 \mathrm{mg}$ of AQ on days 2 and 3 (Guilin Pharmaceutical, Shanghai, China). In addition, they were assigned to receive either $200 \mathrm{mg}$ of AZ or matching placebo on days 1, 2, and 3 (Cipla, Mumbai, India) through double-blinded administration by trial staff [4]. In the village Pê, children did not receive 
prophylactic anti-malarials. For all participants, an artemether-lumefantrine treatment was provided when malaria was diagnosed.

\section{Blood collection}

At the end of each of the eight rounds of drug administration sessions (four sessions per year for two years) and in each of the four villages included in the SMC + AZ trial, 12 to 18 children were randomly selected for blood collection between 14 and 21 days after the start of the administration of prophylactic drugs. Evaluating the effect of chemoprophylaxis on man-to-mosquito transmission in the time frame of two to three weeks after the treatment, while children receive a treatment every four weeks, is a reflection of the effect of drug administration over the whole treatment season. In parallel to the eight treatment sessions, at least 25 untreated children of the control village were randomly selected from census list. For each selected child, $3 \mathrm{ml}$ of blood were collected into a heparinized tube and kept at $37{ }^{\circ} \mathrm{C}$ in an incubator (ENKAB MODEL 70) for transportation to the laboratory in Bobo Dioulasso for $2-3 \mathrm{~h}$ before mosquito feeding. On arrival in the laboratory, thick blood smears were realized using $5 \mu \mathrm{l}$ of blood.

\section{Microscopy}

Thick blood smears were stained with Giemsa (Quimica Clinica Aplicada 990939) and independently examined by three experienced microscopists. Gametocytaemia was based on a count of the number of gametocytes per 1000 leukocytes, and asexual parasitaemia on the count of trophozoites and schizonts against 500 leukocytes, assuming an average number of 8000 leukocytes per $\mu \mathrm{l}$ of blood.

Criteria for concordance and determination of a final result were that after the three reports, results were compared two by two using $\mathrm{V}$, the percentage of concordance: $V=2|A-B| /(A+B)$ where A represents the parasite density reported by reader $1, \mathrm{~B}$ reader 2 . If, $\mathrm{V}$ was $<30 \%$, the parasite density was calculated as the geometric mean of the two values. If $\mathrm{V}$ was $>30 \%$, the third reading was used to do the same calculation with $\mathrm{A}$ and $\mathrm{B}$. Where $\mathrm{A}+\mathrm{C}$ or $\mathrm{B}+\mathrm{C}$ the value is less than $30 \%$, the reading was validated.

\section{Mosquito membrane feeding assay}

A direct mosquito feeding assay procedure (DMFA) previously described [36], was used to estimate drug effect on P. falciparum gametocyte infectivity and mosquito life history traits. From each blood sample, approximately $500 \mu \mathrm{l}$ of blood in heparinized tubes was distributed to each of two membrane feeders and maintained at $37{ }^{\circ} \mathrm{C}$ by circulating water [36]. A mosquito colony of An. gambiae was used. The colony was established in 2008 from wild-caught gravid females collected in Soumousso (40 km southeast of Bobo-Dioulasso) and repeatedly replenished with $\mathrm{F} 1$ from wild-caught mosquito females collected in the same the village. Before the exposure to a blood meal, 3-5 days old female mosquitoes were kept without sucrose solution for $24 \mathrm{~h}$. For each blood sample, two cups each containing 40 female mosquitoes were placed under the feeders to allow blood feeding through parafilm membranes for 30 min. Partially fed and unfed mosquitoes were discarded. Fed mosquitoes were kept in cages $(30 \times 30 \times 30 \mathrm{~cm})$ in the insectary with constant access to $5 \%$ glucose solution on cotton wool pads. Among 550 participants from whom blood could be collected, blood samples obtained from seven subjects could not be used for DMFA for logistics reasons (time between blood collection and exposure to mosquitoes above $4 \mathrm{~h}$ or lack of mosquitoes). On day 7 after membrane feeding, the midguts of mosquitoes of all surviving females were dissected and stained with $0.4 \%$ mercurochrome in phosphate buffered saline (PBS), $\mathrm{pH}$ 7.2. The presence and number of oocysts was recorded for each mosquito by microscopy. The success of mosquito infection was estimated by determining both oocyst prevalence (proportion of $P$. falciparum-infected mosquitoes) and density of infection (mean number of oocysts among infected mosquitoes).

\section{Mosquito life history traits}

The effect of SPAQ and AZ on the blood-feeding rate and survival was investigated. Because the amount of blood ingested can influence the fecundity and survival of the mosquito, blood meal size was considered as a parameter of mosquito life trait. For this purpose, approximately 80 female mosquitoes (2 cups), in addition to the mosquitoes dedicated to transmission assays, were fed to estimate their life history traits using blood samples collected from 77 children in the three study groups. The fed mosquitoes were placed individually in 30-ml drosophila plastic tubes and kept in the insectary.

The blood-feeding rate of mosquitoes exposed to a blood meal was determined by calculating the ratio of fed mosquitoes compared to exposed mosquitoes. Mortality among fed mosquitoes was recorded every $8 \mathrm{~h}$ from the day of membrane blood feeding until death of all mosquitoes. Dead mosquitoes were removed from their individual tubes. Each tube was kept at $+4{ }^{\circ} \mathrm{C}$ before estimation of blood meal size. The size of the blood meal was measured by quantification of haematin, a product of digestion of haemoglobin and 
excreted in faeces of fed mosquitoes. The quantity of excreted haematin was estimated by adding $1 \mathrm{ml}$ of $1 \%$ lithium carbonate (LiCO3) to individual tubes to elute faeces and the absorbance of the resulting solution was read at $387 \mathrm{~nm}$ in a Thermo Scientific Multiskan colorimeter. A LiCO3 solution was used as a blank. Absorbances were compared with a standard curve made with porcine serum haematin to obtain a relative measure of blood meal sizes [37].

\section{Statistical analysis}

All statistical analyses were performed in $\mathrm{R}$ (version 3.4.0). Logistic regression by generalized linear mixed models (GLMM, binomial errors, logit link; lme4 package) was used to investigate the effect of treatment on: (i) asexual parasite prevalence; (ii) gametocyte prevalence; (iii) oocyst prevalence, (proportion of dissected mosquitoes with at least one oocyst in the midgut); and, (iv) mosquito blood-feeding rate. GLMM with negative binomial errors (lme4 package, glmer.nb function) [38] was used to test the effect of treatment on: (i) asexual parasitaemia; (ii) gametocytaemia in participants; and, (iii) oocyst density in mosquitoes. Because blood samples were not fully independent (some participants were sampled multiple times over time, i.e., pseudo-replication), participant identity was included as a random effect in all GLMMs. The effect of treatment on the proportion of infectious feeds was analysed using a binomial GLM. An ANOVA was used to explore the effect of treatment on mosquito blood meal size following a log transformation of haematin concentration. Finally, the effect of treatment on mosquito survivorship was analysed using a mixed effect Cox's proportional hazard regression models (coxme package). Model simplification used stepwise removal of terms, followed by likelihood ratio tests (LRT). Term removals that significantly reduced explanatory power $(\mathrm{p}<0.05)$ were retained in the minimal adequate model [39]. Multiple pairwise post-hoc tests were performed using the package 'Multcomp'.

\section{Results}

Effect of treatment on the human parasite population A total of 550 blood collections were obtained from children aged 24-59 months. Among them, 373 had received chemoprevention (183 SPAQ and 190 SPAQ+AZ) (Fig. 1). The average time between drug administration and blood collection was similar in the two treatment

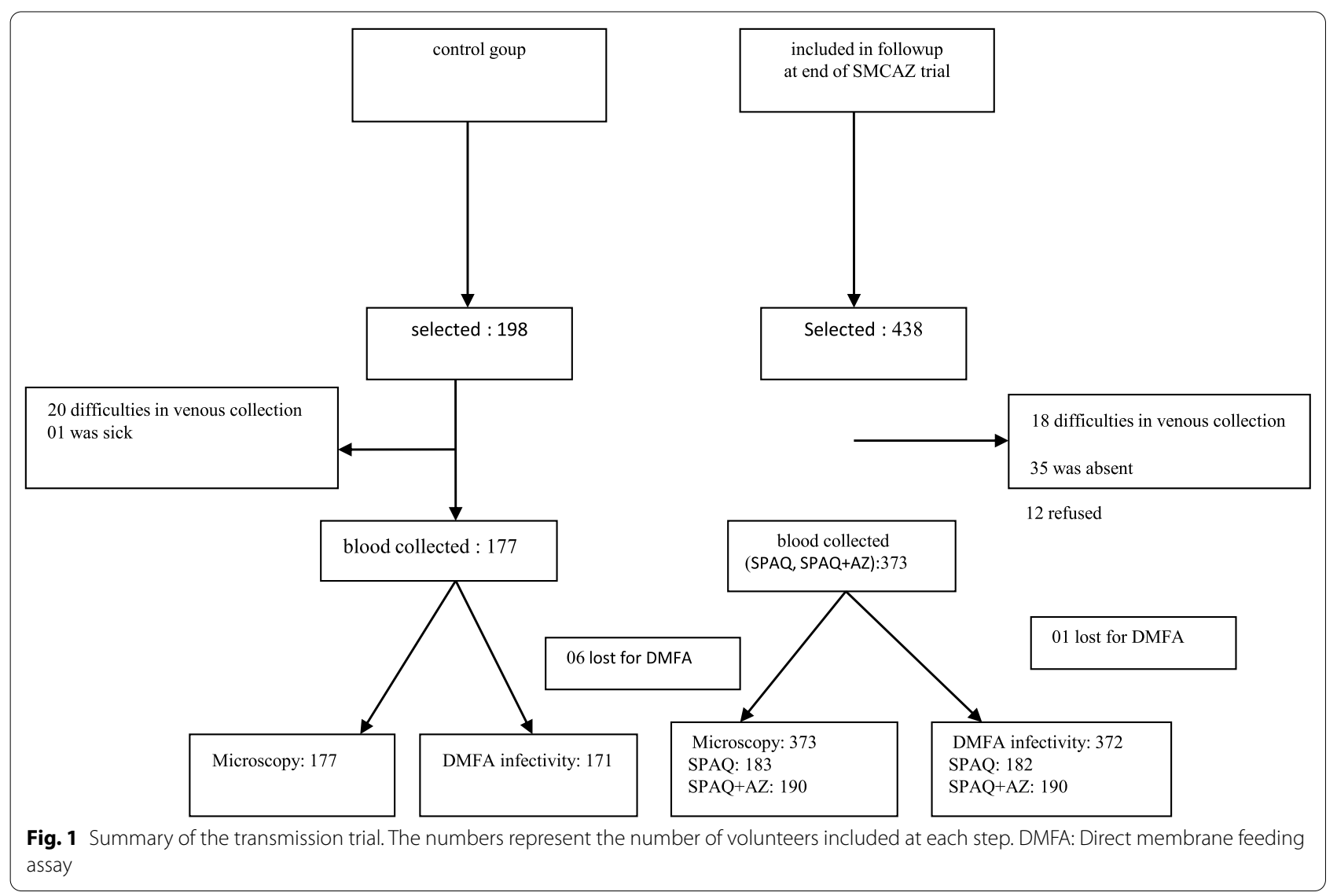



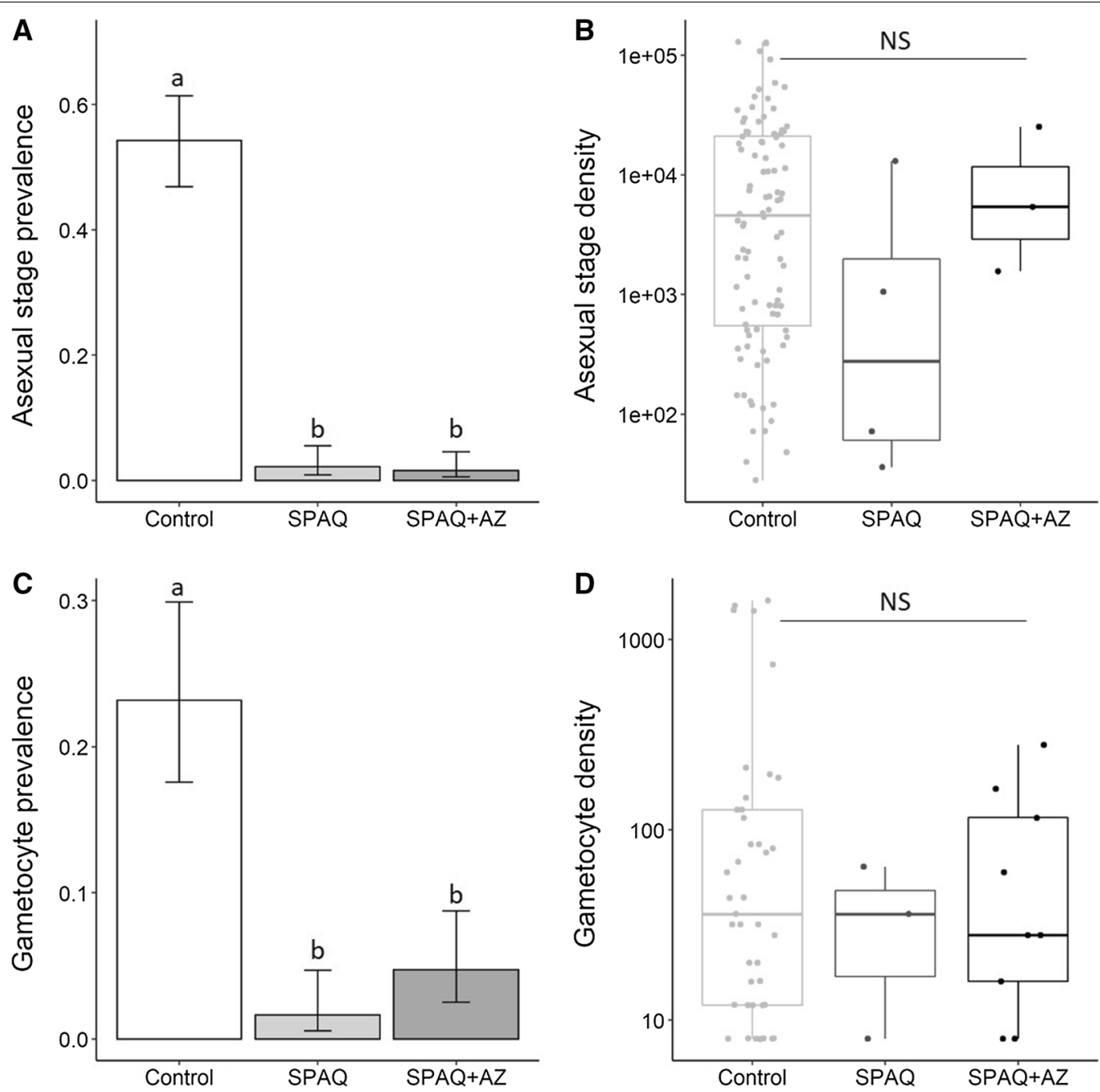

Fig. 2 Effects of SPAQ and SPAQ $+A Z$ on Plasmodium falciparum infection in humans. A The prevalence $( \pm 95 \% \mathrm{Cl})$ of asexual stage parasitaemia for each treatment and the control group. B The density of asexual stage parasites (number of parasites/ $\mathrm{\mu l}$ of blood in infected blood collections) for each treatment and the control group. Each point represents a blood sample from P. falciparum-positive slides. The horizontal line represents the median value of parasite density for each of the three groups, and the upper and lower boundaries of the box indicate the 75th and 25th percentile, respectively. Note that the $y$-axis is on a $\log 10$ scale. C The prevalence $( \pm 95 \% \mathrm{Cl})$ of gametocyte for each treatment and the control group as measured by microscopy; D The density of gametocyte (number of gametocytes/ $\mu$ l of blood in gametocyte-positive blood collections) for each group. Note that the $y$-axis is on a $\log _{10}$ scale. The presence and number of asexual stages or gametocytes in samples was determined by microscopic observation. Different lowercase letters above the bars denote statistically significant differences based on multiple pair-wise post-hoc tests. NS: Not significant

groups, with 16.65 and 16.43 days for the SPAQ and $\mathrm{SPAQ}+\mathrm{AZ}$ groups, respectively $(\mathrm{W}=15,626, \mathrm{P}=0.35)$. The samples were not fully independent as some participants were tested more than once; two participants were sampled three times and 35 other participants were sampled twice. The proportion of children with slides positive for asexual stages of Plasmodium $s p$ in the control group was $55.9 \% \pm 7 \%$ (99/177). Among the 99 positives slides, 96 were positive for $P$. falciparum (97\%), including 9 co-infections with Plasmodium malariae, 6 with Plasmodium ovale and 1 triple infection P. falciparum,
$P$. ovale and $P$. malariae. There were only two monoinfections of $P$. malariae and one mono-infection of $P$. ovale. In the SPAQ treatment group, there were 4/183 (2.19\%) slides positive for P. falciparum asexual stages. In the SPAQ + AZ treatment group 3/190 (1.58\%) slides were positive. The treatments SPAQ and SPAQ + AZ significantly reduced the prevalence of asexual $P$. falciparum (LRT $\mathrm{X}_{2}{ }_{2}=69, \mathrm{P}<0.0001$, Fig. $2 \mathrm{~A}$ ).

There was no difference in P. falciparum asexual prevalence between SPAQ and SPAQ + AZ groups (multiple pairwise comparison: $\mathrm{z}=0.4, \mathrm{P}=0.9$ ). Among infected 

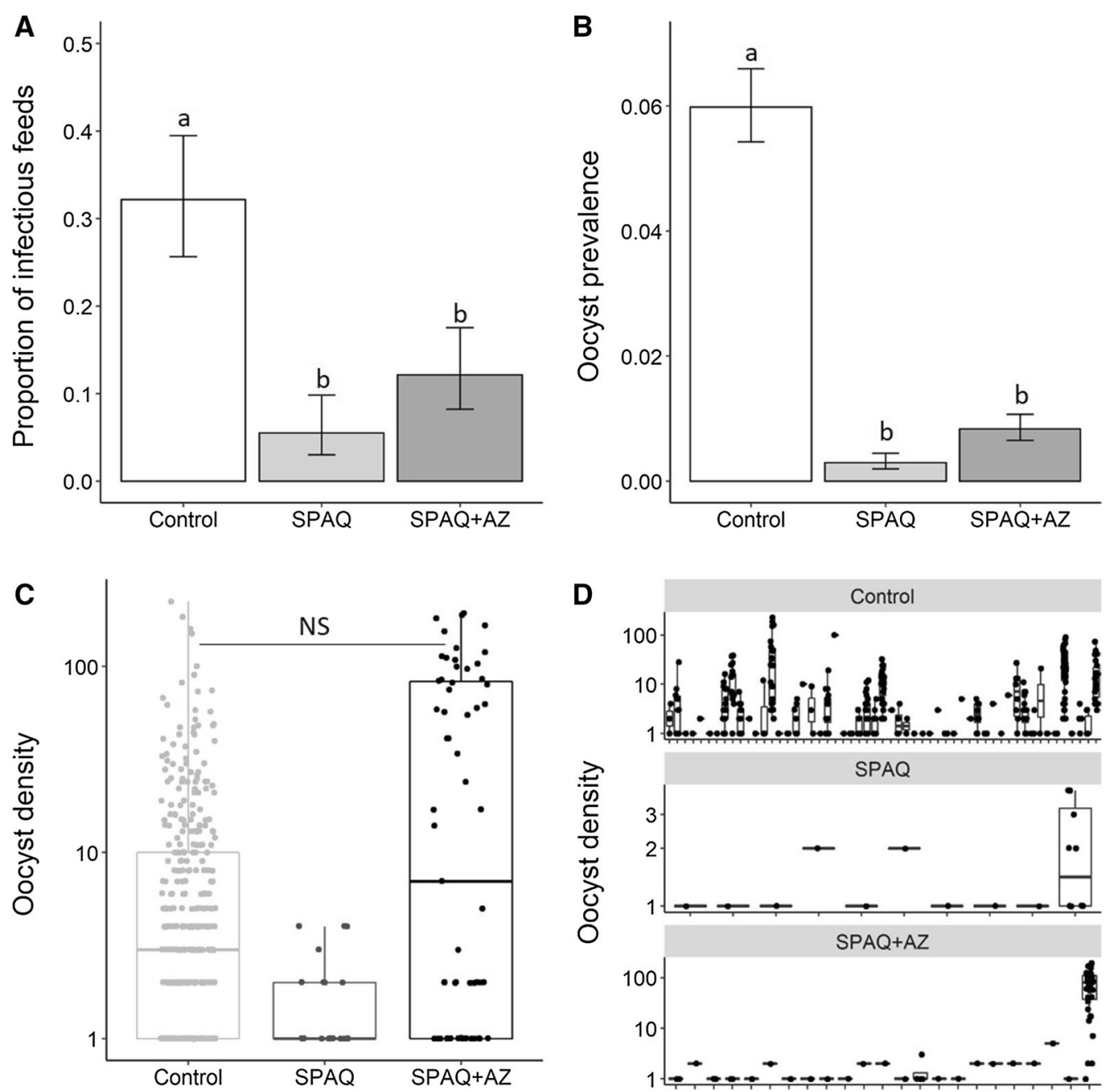

Fig. 3 Effects of SPAQ and SPAQ + AZ on Plasmodium falciparum infectivity from humans to mosquitoes. A The proportion ( $\pm 95 \% \mathrm{Cl}$ ) of feeds resulting in at least one successful mosquito infection (i.e., a minimum of one mosquito harbouring a minimum of one oocyst) for each treatment group. There were 171 feeds for the control, 182 for SPAQ and 190 for the SPAQ-AZ treatment and an average of $38.87 \pm 0.48$ ( median $=41$, range $=5-51$ ) mosquitoes were dissected per feed ( $n$ total $=21,108$ mosquitoes). $\mathbf{B}$ Oocyst prevalence $( \pm 95 \% \mathrm{Cl}$ ) defined as the total number of mosquitoes fed with blood drawn from each study group harbouring at least one oocyst in their midgut out of the number of dissected mosquitoes from this group. C Oocyst density defined as the number of oocysts observed in infected mosquitoes for each group. The horizontal line represents the median value of density for each of the three study groups, and the upper and lower boundaries of the box indicate the 75th and 25th percentile, respectively. D Oocyst density following infectious feeds on samples from the control group, SPAQ group and SPAQ + AZ group. Note that the $y$-axis on panels $(C)$ and $(D)$ is on a $\log _{10}$ scale. Different lowercase letters above the bars denote statistically significant differences based on multiple pair-wise post-hoc tests. NS: Not significant

children in the control group, the mean $P$. falciparum asexual parasite density was $16,074 \pm 2803$ parasites/ $\mu \mathrm{l}$ (range: 28-128,800). The four infected individuals of the SPAQ group had a mean asexual parasitaemia of $3548 \pm 3167$ parasites/ $\mu$ l (range: $36-13,022)$ and among the three positive children from the SPAQ $+\mathrm{AZ}$ group the mean density was $10,709 \pm 7310$ parasites/ $\mu$ l (range: 1560-25,160). Asexual parasite density was not significantly different among the three groups (LRT X ${ }_{2}{ }_{2}=2.27$, $\mathrm{P}=0.32$; Fig. 2B).
Chemoprevention with SPAQ or SPAQ + AZ treatments, significantly reduced the $P$. falciparum gametocyte prevalence (LRT $\mathrm{X}_{2}{ }_{2}=54, \mathrm{P}<0.0001$ ). There were $3 / 183$ (1.6\%) gametocyte-positive slides in the SPAQ group and 9/190 (4.7\%) in the SPAQ + AZ group compared to a prevalence in the control group of $27 \% \pm 6.5$ (48/177). There was no difference in gametocyte prevalence between SPAQ and SPAQ + AZ groups (multiple pairwise comparison: $\mathrm{z}=1.6, \mathrm{P}=0.2$ ). The mean number 
of gametocytes/ $\mu$ l of blood was not influenced by the treatments (LRT X ${ }_{2}^{2}=0.73, \mathrm{P}=0.69$; Fig. 2D).

\section{Effect of treatment on malaria transmission from humans to mosquitoes}

A total of 21.108 mosquitoes, fed with one of 543 blood samples (171 from the control group, 182 from the SPAQ treatment group. and 190 from the SPAQ + AZ treatment group) through direct membrane feeding assays, were dissected seven days post feeding. Mosquitoes were successfully infected from a total of 88 blood samples (55 feeds from the control group, 10 feeds from the SPAQ treatment group and 23 from the SPAQ + AZ treatment group). The proportion of feeds giving a successful mosquito infection varied significantly among the groups (LRT $\mathrm{X}_{2}^{2}=61, \mathrm{P}<0.0001$; Fig. 3A). Thirty-two per cent $(55 / 171)$ of the blood feeds originating from the control group resulted in successful mosquito infection. The proportion of infectious feeds from the SPAQ + AZ group (23/190) was twice as large as that from the SPAQ group (10/182) (LRT X ${ }_{1}^{2}=5.2, \mathrm{P}=0.02$, multiple pair wise comparison: $\mathrm{Z}=2.2, \mathrm{P}=0.07$, Fig. $3 \mathrm{~A}$ ), suggesting a significant effect of $\mathrm{AZ}$ on gametocyte infectivity.

The prevalence of oocysts (i.e., the number of mosquitoes presenting at least one oocyst in the midgut out of the total number of dissected mosquitoes) was higher in the control group than in the two treatment groups
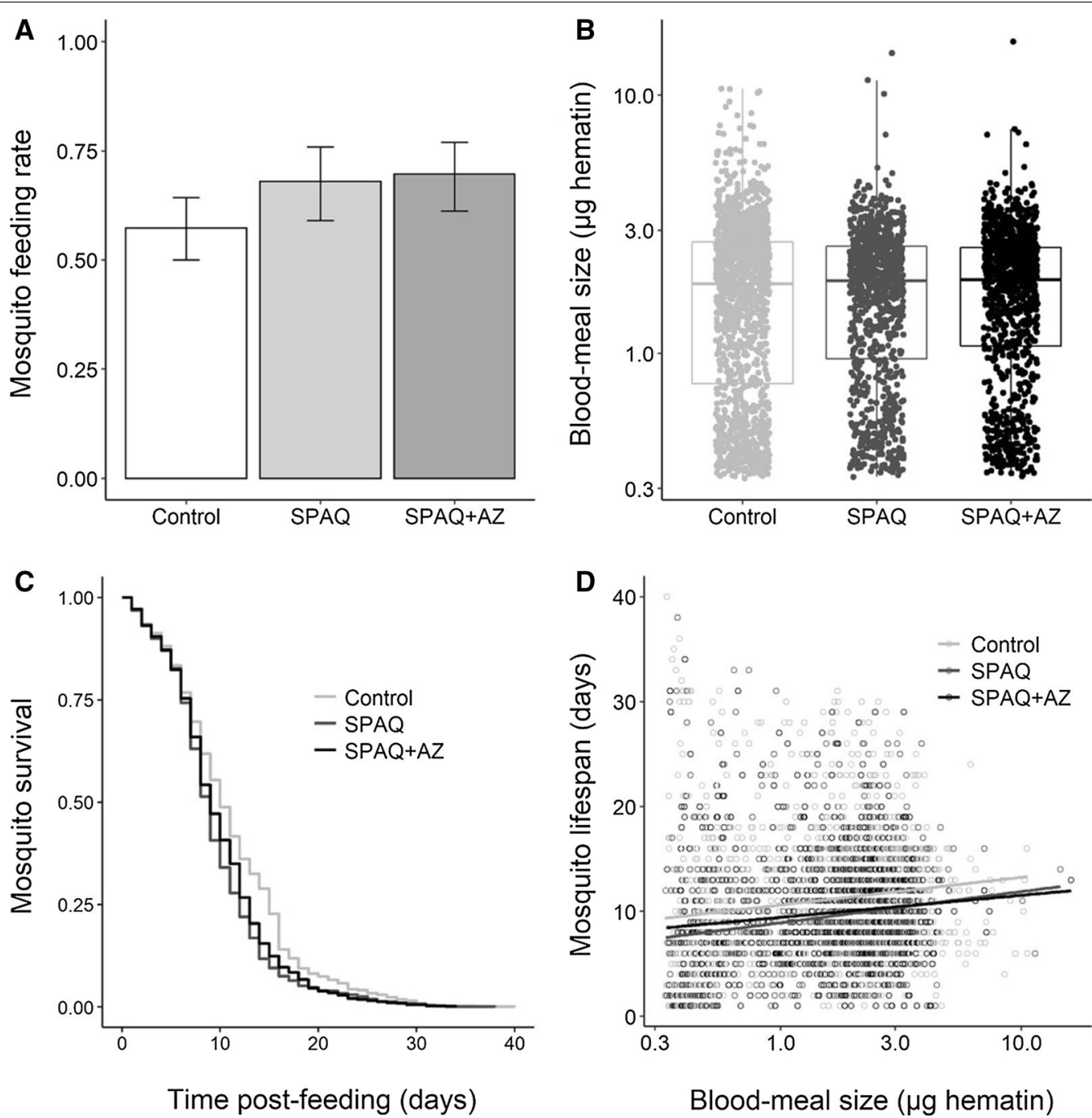

Fig. 4 Effect of SPAQ and SPAQ + AZ on mosquito life history traits. A Mosquito blood-feeding rate, expressed as the proportion (+ 95\% Cl) of engorged mosquitoes out of the total number exposed to the blood meal; B Mosquito blood-meal size, expressed as the quantity of haematin in $\mu \mathrm{g}$ excreted by individual mosquitoes. Note that the $y$-axis is on a $\log _{10}$ scale. C The daily survival of mosquitoes individually kept in drosophila tubes. D The relationship between mosquito lifespan and blood meal size. Note that the $x$-axis is on a $\log _{10}$ scale 
$\left(X_{2}^{2}=22.8, \mathrm{P}<0.001\right.$, Fig. 3B $)$. Following 55 infectious feeds on sample from the control group, including 25 from gametocyte-positive blood samples (19 for $P$. falciparum, 1 for $P$. ovale, 1 for $P$. malariae, 3 mixed infections with $P$. falciparum and $P$. malariae and 1 with $P$. falciparum, $P$. ovale and $P$. malariae), 380 out of 6,349 dissected mosquitoes (6\%), were infected (Fig. 3B). In the SPAQ group, 21 out of 7,207 mosquitoes $(0.29 \%)$ were infected from 10 infectious feeds, including one from a $P$. falciparum gametocyte-positive blood collection (Fig. 3B). In the SPAQ + AZ group, 63 out of 7,552 mosquitoes $(0.8 \%)$ were infected from 23 infectious feeds, including 3 from $P$. falciparum gametocyte-positive blood samples (Fig. 3B). There was no difference in oocyst prevalence between SPAQ and SPAQ+AZ groups (multiple pair wise comparison: $\mathrm{z}=1.3, \mathrm{P}=0.4$ ).

Although mosquitoes infected from blood samples obtained from the SPAQ + AZ group harboured relatively more oocysts in their midguts (mean \pm se density of $43 \pm 7$ ) compared to mosquitoes infected from samples obtained from the control $(10.9 \pm 1.2)$ or SPAQ $(1.8 \pm 0.3)$ groups, the difference was not statistically significant (LRT $X_{2}^{2}=4.4, \mathrm{P}=0.11$, Fig. $3 \mathrm{C}$ ). This is because more than half of the mosquito infections in the SPAQ $+A Z$ group (34/63) came from a single gametocyte carrier. Figure 3D shows the oocyst density produced by each infectious feed $(\mathrm{n}=55$ for the control group, 10 for SPAQ and 23 for SPAQ + AZ). Most infectious feeds from the SPAQ or SPAQ + AZ group resulted in single infected mosquitoes harbouring very few oocysts (Fig. 3D).

\section{Effect of treatment on mosquito blood-feeding success, mosquito blood-meal size and on mosquito survival}

To investigate the effect of treatment on mosquito blood-feeding success, the proportion of fully engorged females was recorded for 34 feeds on blood samples from the control group (2547 mosquitoes), 19 feeds from the SPAQ group (1,491 mosquitoes) or 21 feeds from the SPAQ + AZ group (1647 mosquitoes). A marginally significant effect of treatment on mosquito feeding rate was observed ( $\operatorname{LRT} X^{2}{ }_{2}=6.0, \mathrm{P}=0.048$, Fig. $4 \mathrm{~A}$ ), with slightly

Table 1 Risk of mortality (hazard ratio) along with the standard error, $z$ and P-value for each treatment group relative to the control group

\begin{tabular}{llll}
\hline Treatment & Hazard ratio $(\mathbf{s e})$ & $\mathbf{z}$ & P-value \\
\hline SPAQ & $1.486(0.119)$ & 3.34 & 0.0008 \\
SPAQ + AZ & $1.333(0.117)$ & 2.46 & 0.014 \\
\hline
\end{tabular}

lower feeding success with blood samples from the control group.

To investigate the effect of treatment on mosquito blood-meal size and mosquito survival, fully fed females from 35 feeds on blood samples from the controls group (1440 mosquitoes), 21 feeds from the SPAQ group (947 mosquitoes) or 21 feeds from the SPAQ $+A Z$ group (1,055 mosquitoes) were kept individually in drosophila plastic tubes. The size of the blood meal was measured following the haematin dosage procedure. There was no effect of SPAQ or SPAQ + AZ on mosquito blood meal size (LRT $\mathrm{X}_{2}{ }_{2}=3.0, \mathrm{P}=0.12$, Fig. $4 \mathrm{~B}$ ).

The daily mortality of individual female mosquitoes was recorded. There was a slight but statistically significant negative effect of SPAQ and SPAQ $+\mathrm{AZ}$ on mosquito survival compared to mosquitoes fed with blood samples from the control group (median longevity of mosquitoes 10, 9 and 9 days for the control, SPAQ and SPAQ + AZ groups, respectively, $\mathrm{LRTX}^{2}{ }_{2}=330, \mathrm{P}<0.0001$, Fig. $4 \mathrm{C}$, Table 1, multiple pair-wise comparisons, control-SPAQ: $\mathrm{z}=3.338, \quad \mathrm{P}=0.002, \quad$ control-SPAQ $+\mathrm{AZ}: \quad \mathrm{z}=2.462$, $\mathrm{P}=0.036$, SPAQ-SPAQ + AZ: $\mathrm{z}=0.8, \mathrm{P}=0.7)$.

There was a significant positive relationship between mosquito lifespan and blood meal size (LRT $X^{2}{ }_{1}=142$, $\mathrm{P}<0.001$, Fig. 4D), with females that took large blood meals living longer than those that took smaller blood meals. This relationship was true for all three groups (treatment by blood meal size interaction: LRT $X^{2}{ }_{2}=1.5$, $\mathrm{P}=0.17$, Fig. 4D).

\section{Discussion}

The present study investigated the effect of SMC with or without the addition of $\mathrm{AZ}$ on transmission of malaria. A significant reduction in malaria prevalence was found among blood samples obtained from treated children (SPAQ or SPAQ + AZ) compared to samples from the control group but no significant difference was seen between the SPAQ and SPAQ + AZ groups. This finding indicates that addition of $\mathrm{AZ}$ does not influence the malaria prevalence in the context of SMC as previously observed [4]. Consistently, the prevalence of gametocytes was significantly lower in children treated with SPAQ or $\mathrm{SPAQ}+\mathrm{AZ}$ than in the controls but there was no difference between SPAQ and SPAQ + AZ groups. These findings indicate that SMC with SPAQ may have an effect on the overall transmission of malaria, especially if SMC was extended to older children, but that addition of $\mathrm{AZ}$ will not enhance this effect.

The proportion of mosquitoes infected from blood samples obtained from children in the control group was significantly higher than that in mosquitoes fed on blood from children in the SPAQ or SPAQ + AZ groups. The low rate of infectivity of post-treatment patients may be 
due to the cumulative effects of the treatment; that the sporontocidal activity of SP is known to affect parasite development prior to the stage of mature oocyst when SP is ingested together with gametocytes during the mosquito blood meal $[12,36]$ and the rapid action of AQ on asexual forms that limits the presence and number of asexual parasites that engage in the gametocytogenesis $[15,40,41]$.

A higher proportion of mosquitoes fed on blood samples from children in the SPAQ + AZ group were infectious compared to feeds on samples from children in the SPAQ group suggesting that mosquitoes could be more prone to become infected with Plasmodium when feeding on blood containing AZ. Two hypotheses may explain the present result. Firstly, despite the relatively short halflife of AZ (44 h) [42], AZ remains detectable for more than 20 days, especially in white blood cells [43] and AZ ingested by mosquitoes during blood feeding may increase mosquito permissiveness to malaria infection by affecting their midgut microbiota and subsequently impact on microbe-parasite interactions, as previously observed with other antibiotics $[19,27]$. However, this contrasts with previous findings, which showed that AZ added to an infectious blood meal reduced the parasite load in mosquitoes [28]. Dose-dependent effects of antibiotics on malaria transmission could account for these different findings. Secondly, mechanisms occurring in the human host could explain the influence of $\mathrm{AZ}$ on infectivity of gametocytes to mosquitoes. Because gametocyte density in infected individuals is often too low to be detected by standard light microscopy [44, 45], it cannot be excluded that the AZ treatment increases the prevalence of gametocyte carriage and subsequently increases transmission despite low densities of sexual parasites. Molecular detection and quantification of gametocytes may help understanding the effect of $\mathrm{AZ}$ administration on malaria transmission.

A marginal effect of the treatment with SPAQ or $\mathrm{SPAQ}+\mathrm{AZ}$ on the mosquito feeding rate was observed. Mosquitoes seemed to be more prone to complete a blood meal when blood was collected from children who had received treatment compared to controls. This suggests that SPAQ or SPAQ + AZ influence mosquitoes feeding behaviour, increasing appetite. This may be due to the presence of drug metabolites still present in the blood. Indeed, it has been demonstrated that several chemical families may influence the attraction and feeding behaviour of the vector mosquitoes [46].

Transmission of Plasmodium is closely dependent on the ability of the mosquito to harbour the parasite and to survive long enough for them to develop. The probability of mosquito survival was significantly lower in the treated groups (SPAQ, SPAQ+ AZ) than in the control group, with a median longevity one day shorter, suggesting that the drugs reduced mosquito survival in accordance with previous observations $[17,28]$. Although the observed reduction of survival is moderate in this study, probably due to low concentrations of drugs in blood collected 14 to 21 days post treatment, the reduction of mosquito life span could significantly disturb the Plasmodium sporogonic cycle. Indeed, a decreased mosquito survival rate strongly affects vectorial capacity, by decreasing the likelihood of infected mosquitoes surviving beyond the parasite extrinsic incubation period (time required between being ingested and becoming infective to humans), and therefore the probability of transmitting the disease to new hosts [47].

The moderate lethal effect of the molecules on the mosquito feeding rate and on mosquito longevity would have been more significant if the blood had been drawn in at an interval of time less than 14 days when the concentration of the molecules is higher in the blood. Therefore, subsequent studies could be carried out by taking blood at different times points after the treatment to determine the relation between the time of chemoprevention administration and it effect on mosquito fitness and parasite transmission.

\section{Conclusion}

This study, conducted in Burkina Faso, showed that SMC greatly reduces the infectious parasite reservoir, decreases gametocyte carriage and infectivity to mosquitoes. The finding indicates that the addition of AZ to SPAQ prophylaxis slightly influences human to mosquito malaria transmission, with an increased proportion of infectious mosquito feeds 2 to 3 weeks after the start of treatment in children with AZ or SPAQ. Despite the fact that this effect was marginal and could be due to chance, this is a potentially undesirable effect of adding AZ to SMC and does not support mass administration of this antibiotic in the context of malaria transmission.

The result also showed that SMC using SPAQ, with or without AZ, affects the mosquito's life traits with a slightly enhanced blood feeding success and a decrease in mosquito survival rate. The importance of mosquito longevity in malaria transmission suggests that, between these two effects on mosquito's life traits, the effect on survival may be the most important in terms of vectorial capacity. This suggests that in addition to the prophylactic effect in humans and the reduction of transmission to mosquitoes, SMC may also affect mosquito longevity in a way that confirms its value in the fight against malaria. 
The findings show a strong benefit of SMC in reducing human to mosquito transmission. However, although the effect was only moderate, the results show that the addition of AZ to SPAQ in SMC might favour malaria transmission and does not support mass administration of this drug in the context of malaria control.

\section{Abbreviations}

IPTp: Intermittent preventive treatment during pregnancy; SMC: Seasonal malaria chemoprevention; SP: Sulfadoxine pyrimethamine; AQ: Amodiaquine; AZ: Azithromycin; DMFA: Direct membrane feeding assay; PBS: Phosphate buffered saline; LiCO3: Lithium carbonate; GLMM: Generalized linear mixed models; LRT: Likelihood ratio tests.

\section{Acknowledgements}

We are very grateful to all the participants to the SMC+AZ trial, whom cooperation was essential for the realization of this study. We thank the children and their parents, the personnel of medical centres of Houndé region and the technical staff of the laboratory at IRSS Bobo-Dioulasso.

\section{Authors' contributions}

Conception and study design: KBY, RSY, TL, IZ, AMB, AC, JBO, Data collection analysed: KBY, SBO, YAF, TL, FN, YDC, Experimentation: KBY, RSY, SBO, FAY. Drafting or revising the article the paper: $K B Y, R S Y, D C, B G, A C, J B O$. All authors read and approved the final manuscript.

\section{Funding}

This work was supported by funds from the Institut de Recherche pour le Developpement (IRD) France, through a JEAI Grant, we sincerely thank IRD.

\section{Availability of data and materials}

The data used in this article is available to readers.

\section{Declarations}

\section{Ethics approval and consent to participate}

The article is accepted by the Research Ethics national ethic committee of Burkina Faso under the Registration No. 2015-5-56.

\section{Consent for publication}

All authors discussed the results and commented on the manuscript, and consent for publication.

\section{Competing interests}

We declare that no competing interests existed for the authors or the institutes before, during and after preparing and submitting this paper for review.

\begin{abstract}
Author details
${ }^{1}$ Institut de Recherche en Sciences de la Santé, Bobo-Dioulasso, Burkina Faso. ${ }^{2}$ Université Nazi Boni, Bobo-Dioulasso, Burkina Faso. ${ }^{3}$ Institut des Sciences et Techniques (INSTech Bobo), BP2779 Bobo-Dioulasso, Burkina Faso. ${ }^{4}$ MIVEGEC, University of Montpellier, IRD, CNRS, Montpellier, France. ${ }^{5}$ Laboratoire Mixte International Sur Les Vecteurs (LAMIVECT), Bobo Dioulasso, Burkina Faso. ${ }^{6}$ Centre de Recherche en Écologie et Évolution de la Santé (CREES), Montpellier, France. ${ }^{7}$ Institut de Recherche en Sciences de la Santé, Nanoro, Burkina Faso. ${ }^{8}$ London School of Hygiene and Tropical Medicine, London, UK.
\end{abstract}

Received: 23 April 2021 Accepted: 16 July 2021

Published online: 27 July 2021

\section{References}

1. WHO. World malaria report 2020. Geneva: World Health Organization; 2020
2. WHO Policy Recommendation. Seasonal Malaria Chemoprevention (SMC) for Plasmodium falciparum malaria control in highly seasonal transmission areas of the Sahel sub-region in Africa. Geneva: World Health Organization; 2012.

3. Partnership A. Effectiveness of seasonal malaria chemoprevention at scale in west and central Africa: an observational study. Lancet. 2020;396:1829-40.

4. Chandramohan D, Dicko A, Zongo I, Sagara I, Cairns M, Kuepfer I, et al. Effect of adding azithromycin to seasonal malaria chemoprevention. N Engl J Med. 2019;380:2197-206.

5. Porco TC, Gebre T, Ayele B, House J, Keenan J, Hong KC, et al. Effect of mass distribution of azithromycin for trachoma control on overall mortality in Ethiopian children : a randomized trial. JAMA. 2009;302:962-8.

6. Keenan JD, Ayele B, Gebre T, Zerihun M, Zhou Z, House Jl, et al. Childhood mortality in a cohort treated with mass azithromycin for trachoma. Clin Infect Dis. 2011;52:883-8.

7. Talman AM, Domarle O, Mckenzie FE, Ariey F, Robert V. Gametocytogenesis: the puberty of Plasmodium falciparum. Malar J. 2004;3:24.

8. Bousema T, Drakeley C. Epidemiology and infectivity of Plasmodium falciparum and Plasmodium vivax gametocytes in relation to malaria control and elimination. Clin Microbiol. 2011;24:377-410.

9. Hobbs CV, Tanaka TQ, Muratova O, Van VJ, Borkowsky W, Williamson KC, et al. HIV treatments have malaria gametocyte killing and transmission blocking activity. J Infect Dis. 2013;208:139-48.

10. Dechy-Cabaret O, Benoit-Vical F. Effects of antimalarial molecules on the gametocyte stage of Plasmodium falciparum: the debate. J Med Chem. 2012:15:10328-44.

11. Delves M, Plouffe D, Scheurer C, Meister S, Wittlin S, Winzeler EA, et al. The activities of current antimalarial drugs on the life cycle stages of Plasmodium: a comparative study with human and rodent parasites. PLoS Med. 2012;9:e1001169.

12. Robert V, Awono-ambene HP, Hesran EJL. Gametocytemia and infectivity to mosquitoes of patients with uncomplicated Plasmodium falciparum malaria attacks treated with chloroquine or sulfadoxine plus pyrimethamine. Am J Trop Med Hyg. 2000;62:210-6.

13. Puta C, Manyando C. Enhanced gametocyte production in Fansidartreated Plasmodium falciparum malaria patients: implications for malaria transmission control programmes. Trop Med Int Health. 1997;2:227-9.

14. Sokhna C, Trape J, Robert V. Gametocytaemia in senegalese children with uncomplicated P. falciparum malaria treated with chloroquine, amodiaquine or sulfadoxine + pyrimethamine. Parasite. 2001;8:243-50.

15. Kiszewski EA. Blocking Plasmodium falciparum malaria transmission with drugs: the gametocytocidal and sporontocidal properties of current and prospective antimalarials. Pharmaceuticals. 2011;4:44-68.

16. Beavoguia AH, Djimde A, Gregsonb A, Toure MA, Dao A, Coulibaly B, et al. Low infectivity of Plasmodium falciparum gametocytes to Anopheles gambiae following treatment with sulfadoxine- pyrimethamine in Mali. Int J Parasitol. 2010;40:1213-20.

17. Kone A, van de Vegte-Bolmer M, Siebelink-Stoter R, van Gemert GJ, Dara A, Niangaly $\mathrm{H}$, et al. Sulfadoxine-pyrimethamine impairs Plasmodium falciparum gametocyte infectivity and Anopheles mosquito survival. Int J Parasitol. 2010;40:1221-8.

18. Pumpuni C, Demaio J, Kent M, Davis J, Beier J. Bacterial population dynamics in three anopheline species: the impact on Plasmodium sporogonic development. Am J Trop Med Hyg. 1996;54:214-8.

19. Boissiere A, Tchioffo M, Bachar D, Abate L, Marie A, Boissie A, et al. Midgut microbiota of the malaria mosquito vector Anopheles gambiae and interactions with Plasmodium falciparum infection. PLoS Pathog. 2012;8:e1002742.

20. Tchioffo MT, Boissiere A, Churcher TS, Abate L, Gimonneau G, Christen R, et al. Modulation of malaria infection in Anopheles gambiae mosquitoes exposed to natural midgut bacteria. PLoS One. 2013;8:e81663.

21. Guégan M, Zouache K, Démichel C, Minard G, Van VT, Potier P, et al. The mosquito holobiont: fresh insight into mosquito-microbiota interactions. Microbiome. 2018;6:49.

22. Weiss B, Aksoy S. Microbiome influences on insect host vector competence. Trends Parasitol. 2011;27:514-22.

23. Dennison NJ, Saraiva RG, Cirimotich CM, Mlambo G, Mongodin EF, Dimopoulos G. Functional genomic analyses of Enterobacter, Anopheles and Plasmodium reciprocal interactions that impact vector competenc. Malar J. $2016: 15: 425$. 
24. Cirimotich CM, Dong Y, Clayton AM, Sandiford SL, Souza-Neto JA, Mulenga $M$, et al. Natural microbe-mediated refractoriness to Plasmodium infection in Anopheles gambiae. Science. 2011;332:855-8.

25. Angleró-rodríguez Yl, Blumberg BJ, Dong Y, Sandiford SL, Pike A, Clayton AM, et al. A natural Anopheles -associated Penicillium chrysogenum enhances mosquito susceptibility to Plasmodium infection. Sci Rep. 2016;6:34084

26. Stathopoulos S, Neafsey DE, Lawniczak MKN, Muskavitch MAT, Christophides GK. Genetic dissection of Anopheles gambiae gut epithelial responses to Serratia marcescens. PLoS Pathog. 2014;10:e1003897.

27. Gendrin M, Rodgers FH, Yerbanga SR, Ouedraogo JB, Basanez M-G, Cohuet $A$, et al. Antibiotics in ingested human blood affect the mosquito microbiota and capacity to transmit malaria. Nat Commun. 2015;6:5921.

28. Gendrin M, Yerbanga RS, Ouedraogo JB, Lefèvre T, Cohuet A, Christophides GK. Differential effects of azithromycin, doxycycline, and cotrimoxazole in ingested blood on the vectorial capacity of malaria mosquitoes. Open Forum Infect Dis. 2016;3:ofw074.

29. Delves MJ, Ruecker A, Straschil U, Lelièvre J, Marques S, López-barragán MJ. Male and female Plasmodium falciparum mature gametocytes show different responses to antimalarial drugs. Antimicrob Agents Chemother. 2013;57:3268-74

30. Dabiré KR, Diabaté A, Namountougou M, Toé KH, Ouari A, Kengne P, et al. Distribution of pyrethroid and DDT resistance and the L1014F kdr mutation in Anopheles gambiae s.I. from Burkina Faso (West Africa). Trans R Soc Trop Med Hyg. 2009;103:1113-20.

31. Namountougou M, Baldet T, Diabate A, Martin T, Dabire RK. Multiple insecticide resistance in Anopheles gambiae s.l. populations from Burkina Faso, West Africa. PLoS One. 2012;7:48412.

32. Cairns ME, Sagara I, Zongo I, Irene K, Ismaila T, Nikiema F, et al. Evaluation of seasonal malaria chemoprevention in two areas of intense seasonal malaria transmission: secondary analysis of a household-randomised, placebo-controlled Trial in Houndé District, Burkina Faso and Bougouni District, Mali. PLoS Med. 2020;17:e1003214.

33. Ministere de la santé du Burkina-Faso. Annuaire statistique 2013-2014

34. Ministère de l'Economie et des Finances du Burkina-Faso. Cinquième Recensement Général de la Population et de l'Habitation du Burkina Faso: résultats préliminaires; 2020.

35. Ministère de la Santé du Burkina Faso. Carte sanitaire 2010. Burkina-Faso; 2012
36. Gouagna L, Yao F, Yameogo B, Dabiré RK. Comparison of field-based xenodiagnosis and direct membrane feeding assays for evaluating host infectiousness to malaria vector Anopheles gambiae. Acta Trop. 2014;130:131-9.

37. Sangare I, Michalakis Y, Yameogo B, Dabire R, Morlais I, Cohuet A. Studying fitness cost of Plasmodium falciparum infection in malaria vectors: validation of an appropriate negative control. Malar J. 2013;12:2.

38. Bates $\mathrm{D}$, Maechler M, Bolker B, Walker S. Conditional model selection in mixed-effects models with cAIC4. J Stat Softw. 2015:67:1-48.

39. Crawley M. The R Book. 1st edt. Wiley, UK; 2007.

40. Bruce MC, Alano P, Duthie S, Carter R. Commitment of the malaria parasite Plasmodium falciparum to sexual and asexual development. Parasitology. 1990;100:191-200.

41. Baker DA. Malaria gametocytogenesis. Mol Biochem Parasitol. 2010;172:57-65.

42. Tabbara KF, Al-Kharashi SA, Al-Mansouri SM, Al-Omar OM, Cooper H. Foulds AMAE-AG Ocular levels of azithromycin. Arch Ophthalmol. 1998:116:1625-8.

43. Dumitrescu TP, Brouwer KLR, Schmith VD. Population pharmacokinetics of azithromycin in whole blood, peripheral blood mononuclear cells, and polymorphonuclear cells in healthy adults. CPT Pharmacometrics Syst Pharmacol. 2014;3:e103.

44. Ouedraogo AL, Bousema T, Schneider P, De Vlas SJ, Ilboudo-sanogo E, Cuzin-ouattara N, et al. Substantial contribution of submicroscopical Plasmodium falciparum gametocyte carriage to the infectious reservoir in an area of seasonal transmission. PLoS One. 2009;4:e8410.

45. Karl S, Laman M, Koleala T, Ibam C, Kasian B, Drewei NN, et al. Comparison of three methods for detection of gametocytes in Melanesian children treated for uncomplicated malaria. Malar J. 2014;13:319.

46. Zwiebela LJ, Takken W. Olfactory regulation of mosquito-host interactions. Insect Biochem Mol Biol. 2004;34:645-52.

47. Brady OJ, Godfray HCJ, Tatem AJ, Gething PW, Cohen JM, Mckenzie FE. Vectorial capacity and vector control: reconsidering sensitivity to parameters for malaria elimination. Trans R Soc Trop Med Hyg. 2016;1 10:107-17.

\section{Publisher's Note}

Springer Nature remains neutral with regard to jurisdictional claims in published maps and institutional affiliations.
Ready to submit your research? Choose BMC and benefit from:

- fast, convenient online submission

- thorough peer review by experienced researchers in your field

- rapid publication on acceptance

- support for research data, including large and complex data types

- gold Open Access which fosters wider collaboration and increased citations

- maximum visibility for your research: over 100M website views per year

At BMC, research is always in progress.

Learn more biomedcentral.com/submissions 